Diabetologe 2012 $\cdot 8: 52-52$

DOI 10.1007/s11428-011-0845-2

Online publiziert: 15. Januar 2012

๑) Springer-Verlag 2012

\title{
B. Nowotny
}

Institut für Klinische Diabetologie, Deutsches Diabetes-Zentrum an der Heinrich-Heine-Universität,

Leibniz-Zentrum für Diabetesforschung, Düsseldorf

\section{Kontinuierliche subkutane Insulintherapie bei diabetischer Gastroparese}

kontinuierlichen subkutanen Insulintherapie und Optimierung der prokinetischen Therapie stationär aufgenommen und über 12 Monate beobachtet. Die Anzahl der stationären Tage wegen Symptomen der Gastroparese oder instabiler Blutzuckereinstellung reduzierte sich von im Mittel 8,5 Tagen (Spanne 0-144 Tage) auf 0 Tage (Spanne 0-15 Tage). Damit verbunden war eine signifikante Verbesserung des $\mathrm{HbA}_{1 \mathrm{c}}$-Wertes auf 8,0\%, und auch die mittleren Blutzuckerspiegel sanken signifikant ab. Die Insulindosis konnte auf $34 \pm 12 \mathrm{U}$ gesenkt werden, der Gewichtsanstieg in den ersten 6 Monaten betrug 2,9 kg.

\section{Kommentar}

Diese Fallserie zeigt eine beeindruckende Reduktion der Krankenhaustage ebenso wie eine verbesserte Blutzuckereinstellung in dieser sehr schwierig zu behandelnden Patientengruppe. Die kontinuierliche Applikation erlaubt eine noch bessere Anpassung an die schwankenden Magenentleerungszeiten und die wechselnde Resorptionskinetik als eine intensivierte Insulintherapie, und möglicherweise führt die Reduktion der Blutzuckerspitzen auch zu einer Verbesserung der Magenmotilität. Diese Studie liefert damit als „Proof-of-principle-Studie“ einen Ausgangspunkt für weitere, randomisierte Untersuchungen, die neben den zugrundeliegenden Mechanismen auch die langfristigen Effekte - Stichwort Gewichtszunahme - untersuchen sollten.

\section{Korrespondenzadresse}

\section{Dr. B. Nowotny}

Institut für Klinische Diabetologie, Deutsches

Diabetes-Zentrum an der Heinrich-Heine-

Universität, Leibniz-Zentrum für

Diabetesforschung

Auf'm Hennekamp 65, 40225 Düsseldorf

bettina.nowotny@ddz.uni-duesseldorf.de

Interessenkonflikt. Die korrespondierende Autorin weist auf folgende Beziehungen hin: Reisekostenübernahme durch Sanofi-Aventis. 